\title{
Noether symmetric classical and quantum scalar field cosmology
}

\author{
${\text { Babak Vakili }{ }^{1 *} \text { and Farhad Khazaie }}^{2}$ \\ ${ }^{1}$ Department of Physics, Chalous Branch of Azad University, P.O. Box 46615-397, Chalous, Iran \\ ${ }^{2}$ Department of Physics, Tehran Central Branch of Azad University, Tehran, Iran
}

\begin{abstract}
We study the evolution of a two dimensional minisuperspace cosmological model in classical and quantum levels by the Noether symmetry approach. The phase space variables turn out to correspond to the scale factor of a Friedmann-Robertson-Walker (FRW) model and a scalar field with which the action of the model is augmented. It is shown that the minisuperspace of such a model is a two dimensional manifold with vanishing Ricci scalar. We present a coordinate transformation which cast the corresponding minisuper metric to a Minkowskian or Euclidean one according to the choices of an ordinary or phantom model for the scalar field. Then, the Noether symmetry of such a cosmological model is investigated by utilizing the behavior of the corresponding Lagrangian under the infinitesimal generators of the desired symmetry. We explicitly calculate the form of the scalar field potential functions for which such symmetries exist. For these potential functions, the exact classical and quantum solutions in the cases where the scalar field is an ordinary or a phantom one, are presented and compared.
\end{abstract}

PACS numbers: 98.80.-k, 98.80.Qc, 04.60.Ds, 04.60.Kz

Keywords: Noether symmetry, Scalar field cosmology, Quantum cosmology

\section{Introduction}

Classical, semiclassical and quantum scalar fields have played a central role in conceptual discussion of unified theories of interactions and also in all branches of the modern cosmological theories. From a cosmological point of view, there is a renewed interest in the scalar-tensor models in which a nonminimal coupling appears between the geometry of space-time and a scalar field [1]. This is because a number of different scenarios in cosmology such as spatially flat and accelerated expanding universe at the present time [2], inflation [3], dark matter and dark energy [4], and a rich variety of behaviors can be accommodated phenomenologically by scalar fields. Traditionally, cosmological models of inflation use a single scalar field with a canonical kinetic term of the form $1 / 2 g^{\mu \nu} \partial_{\mu} \phi \partial_{\nu} \phi$ with some particular self-interaction potential $V(\phi)$ like $1 / 2 m \phi^{2}$ or $\lambda \phi^{4}$, etc. Such a scalar field is often known as minimally coupled to the geometry. However, there are also scalar fields in what is qualified to be called the scalar-tensor theory, which are not simply added to the tensor gravitational field, but comes into play through the non-minimal coupling term [5]. On the other hand, the current cosmological observations allow the possibility of existence of a cosmic fluid with equation of state parameter smaller than -1 [6]. One of the simplest explanations of such an equation of state is considering a scalar field with negative kinetic energy which is called a phantom field [7]. The classical and quantum cosmological dynamics of the phantom scalar fields has been studied in a number of works, see for example [8].

In this paper we shall study the classical and quantum dynamics of a flat FRW model with a scalar field as its source by the Noether symmetry approach. We set up an effective Lagrangian in which the

*b-vakili@iauc.ac.ir 
scale factor $a$ and scalar field $\phi$ play the role of independent dynamical variables. This Lagrangian is constructed in such a way that its variation with respect to $a$ and $\phi$ yields the appropriate equations of motion. The form of the potential function of the scalar field is then found by demanding that the Lagrangian admits the desired Noether symmetry [9]. By the Noether symmetry of a given minisuperspace cosmological model we mean that there exists a vector field $X$, as the infinitesimal generator of the symmetry, on the tangent space of the configuration space such that the Lie derivative of the Lagrangian with respect to this vector field vanishes. In [10] the applications of the Noether symmetry in various cosmological models are studied. Here, before applying the Noether symmetry condition on the model under consideration, we shall see that the corresponding minisuperspace of our model is a two dimensional Riemannian manifold with zero Ricci scalar. Therefore, it is possible to find a new set of coordinates in terms of which the minisuper metric takes the form of a Minkowskian (when the scalar field is an ordinary quintessence field) or Euclidean (when the scalar field is a phantom field) space. The desired coordinate transformation brings the Lagrangian into a canonical form where its kinetic and part of its potential terms are like the ones of a couple of harmonic oscillators. We shall see by demanding the Noether symmetry as a feature of this Lagrangian, we can obtain the explicit form of the potential function. Since the existence of a symmetry results in a constant of motion, we can integrate the field equations which would then lead to expansion law of the universe.

The structure of the article is as follows. In section 2, we briefly present the basic elements of the issue of Noether symmetry. In section 3, we first introduce the scalar field FRW cosmology and write its Lagrangian in terms of the minisuperspace variables. This section is then divided into two subsections each of which will deal with one kind (quintessence or phantom) of scalar field. In these two subsections, after applying the Noether symmetry condition on the model, we provide the analytical solutions for the corresponding Noether symmetric classical and quantum cosmologies. Finally, the conclusions are summarized in section 4.

\section{The Noether symmetry}

In this section we briefly review the issue of the Noether symmetry approach used in the present work which originally appeared in [11]. To do this, we consider a dynamical system with finite degrees of freedom moving in a Riemannian space with the metric components $\mathcal{G}_{A B}$. The evolution of such a system can be produced by the following action

$$
\mathcal{S}=\int_{\mathcal{M}} d \tau\left[\frac{1}{2} \mathcal{G}_{A B} \frac{d q^{A}}{d \tau} \frac{d q^{B}}{d \tau}-\mathcal{U}(\mathbf{q})\right]
$$

where $q^{A}$ are the dynamical variables representing the configuration space of the system (the indices $A$, $B, \ldots$ run over the dimension of this space), $\mathcal{U}(\mathbf{q})$ is the potential function and $\tau$ is an affine parameter along the evolution path of the system. In time parameterized theories, for instance general relativity, in which their action is invariant under time reparameterization, the affine parameter $\tau$ can be linked to a time parameter $t$ by a lapse function $N(t)$ through $N d t=d \tau$. Therefore, for such a dynamical systems the action (1) can be written as

$$
\mathcal{S}=\int_{\mathcal{M}} d t \mathcal{L}\left(q^{A}, \dot{q}^{A}\right)=\int_{\mathcal{M}} d t N\left[\frac{1}{2 N^{2}} \mathcal{G}_{A B} \dot{q}^{A} \dot{q}^{B}-\mathcal{U}(\mathbf{q})\right]
$$

where now an over dot indicates derivation with respect to the time parameter $t$ and $\mathcal{L}(\mathbf{q}, \dot{\mathbf{q}})$ is the Lagrangian function of the system. Variation of the above action with respect to $q^{A}$ yields the equations of motion as

$$
\frac{1}{N} \frac{d}{d t}\left(\frac{\dot{q}^{A}}{N}\right)+\frac{1}{N^{2}} \Gamma_{B C}^{A} \dot{q}^{B} \dot{q}^{C}=F^{A},
$$


where $F^{A}=-\mathcal{G}^{A B} \partial_{B} \mathcal{U}$ is a force term and $\Gamma_{B C}^{A}=\frac{1}{2} \mathcal{G}^{A D}\left(\partial_{B} \mathcal{G}_{D C}+\partial_{C} \mathcal{G}_{B D}-\partial_{D} \mathcal{G}_{B C}\right)$ is the Christoffel symbols associated with the metric $\mathcal{G}_{A B}$. In this formalism $N$ is not a dynamical variable in the sense that variation of the action (2) with respect to it yields

$$
\frac{1}{2 N^{2}} \mathcal{G}_{A B} \dot{q}^{A} \dot{q}^{B}+\mathcal{U}(\mathbf{q})=\mathbf{0}
$$

which is nothing but the Hamiltonian constraint, a constraint that should hold during the evolution of the system and is indeed a reflect of the fact that the underlying theory is a time parameterized theory. Basically, the equations of motion (3) are the geodesic equations of a "point particle" (=the system) moving in a Riemannian space under the act of the potential field $\mathcal{U}(\mathbf{q})$. Quantization of such a dynamical system may be achieved by the method of canonical quantization. For this purpose we introduce the momenta conjugate to the variables $q^{A}$ by

$$
P_{A}=\frac{\partial \mathcal{L}}{\partial \dot{q}^{A}}=\mathcal{G}_{A B} \frac{\dot{q}^{B}}{N}
$$

leading to the following Hamiltonian function

$$
H=P_{A} \dot{q}^{A}-\mathcal{L}=N\left[\frac{1}{2} \mathcal{G}^{A B} P_{A} P_{B}+\mathcal{U}(\mathbf{q})\right]=N \mathcal{H}
$$

Because of the constraint equation (4), the above Hamiltonian seems to be identically equal to zero. Therefore, under canonical quantization this Hamiltonian yields the Wheeler-DeWitt (WDW) equation $\mathcal{H} \Psi(\mathbf{q})=0$, where $\Psi(\mathbf{q})$ is the wave function of the quantized system and $\mathcal{H}$ should be written in a suitable operator form. If one makes a natural choice of factor ordering, the WDW equation may be written as [12]

$$
\mathcal{H} \Psi(\mathbf{q})=\left[-\frac{1}{2} \nabla^{2}+\mathcal{U}(\mathbf{q})\right] \Psi(\mathbf{q})=0
$$

where $\nabla^{2}=\frac{1}{\sqrt{-\mathcal{G}}} \partial_{A}\left(\sqrt{-\mathcal{G}} \mathcal{G}^{A B} \partial_{B}\right)$ is the Laplacian operator in the space with metric $\mathcal{G}_{A B}$.

Now, let us deal with the idea of the Noether symmetry in a given dynamical system like that one presented above. Following [11], we define the Noether symmetry as a vector field $X$ on the tangent space $T Q=(\mathbf{q}, \dot{\mathbf{q}})$ of the configuration space through

$$
X=\alpha^{A}(\mathbf{q}) \frac{\partial}{\partial q^{A}}+\frac{d \alpha^{A}(\mathbf{q})}{d t} \frac{\partial}{\partial \dot{q}^{A}}
$$

where $\alpha^{A}(\mathbf{q})$ are unknown functions on configuration space. The Noether symmetry then implies that the Lie derivative of the Lagrangian with respect to this vector field vanishes, that is, $L_{X} \mathcal{L}=0$, which leads

$$
L_{X} \mathcal{L}=\alpha^{A}(\mathbf{q}) \frac{\partial \mathcal{L}}{\partial q^{A}}+\frac{d \alpha^{A}(\mathbf{q})}{d t} \frac{\partial \mathcal{L}}{\partial \dot{q}^{A}}=0 .
$$

Noether symmetry approach is a powerful tool in finding the solutions to a given Lagrangian, including the one presented above. In this approach, one is concerned with finding the cyclic variables related to conserved quantities and consequently reducing the dynamics of the system to a manageable one. The existence of Noether symmetry means that phase flux is conserved along the vector field $X$ and thus a constant of motion exists. Indeed, noting that $P_{A}=\frac{\partial \mathcal{L}}{\partial \dot{q}^{A}}$ and also taking into account the Euler-Lagrange equations $\frac{d P_{A}}{d t}=\frac{\partial \mathcal{L}}{\partial q^{A}}$, from (9) we get $\frac{d}{d t}\left[\alpha^{A}(\mathbf{q}) P_{A}\right]=0$. Thus the constants of motion are found as

$$
Q=\alpha^{A}(\mathbf{q}) P_{A}
$$


In order to obtain the functions $\alpha^{A}(\mathbf{q})$ we use equation (9). However, in some cases which we prefer to use the Hamiltonian formalism of the theory, the Hamiltonian counterpart of this equation is better suited in finding these coefficients which, equivalently, can be written as

$$
\alpha^{A}\left\{P_{A}, H\right\}+\frac{\partial \alpha^{A}}{\partial q^{B}}\left\{q^{B}, H\right\} P_{A}=0 .
$$

In general, the expression above gives a quadratic polynomial in terms of momenta with coefficients being partial derivatives of $\alpha^{A}$ with respect to the configuration variables $\mathbf{q}$. Thus, the expression is identically equal to zero if and only if these coefficients are zero, leading to a system of partial differential equations for $\alpha^{A}(\mathbf{q})$. An important ingredient in any model theory related to Noether symmetry is the choice of the cyclic variables related to this symmetry. Since such a variable gives a constant of motion, it should have a vanishing commutator with the Hamiltonian and therefore from a quantum mechanical point of view they should have simultaneous eigenfunctions. This means that to describe the quantum structure for a Noether symmetric model, one should take into account the commutative algebra between the Hamiltonian and the conserved quantities (10). If $Q$ is itself a cyclic variable the quantum counterpart of the theory can be described by the following equations

$$
\left\{\begin{array}{l}
\mathcal{H} \Psi(\mathbf{q})=0 \\
\hat{Q} \Psi(\mathbf{q})=\mathcal{Q} \Psi(\mathbf{q}),
\end{array}\right.
$$

where $\hat{Q}$ is the operator form of (10) and $\mathcal{Q}$ is its eigenvalue. If $Q$ is not a cyclic variable this procedure does not work. In this case we seek a change of variables in the form of a point transformation $\mathbf{q}=\left(q^{1}, q^{2}, \ldots\right) \rightarrow \mathbf{u}=\left(u^{1}, u^{2}, \ldots\right)$ on the vector field (8) such that in terms of the new variables $\mathbf{u}$, the Lagrangian includes one (or more) cyclic variable. A general discussion of this issue can be found in [11]. Under such a point transformation it is easy to show that the vector field (8) takes the form [13]

$$
\tilde{X}=\left(X u^{A}\right) \frac{\partial}{\partial u^{A}}+\frac{d}{d t}\left(X u^{A}\right) \frac{\partial}{\partial \dot{u}^{A}} .
$$

it is easy to show that if $X$ is a Noether symmetry, $\tilde{X}$ has also this property, that is, $X \mathcal{L}=0 \Rightarrow$ $\tilde{X} \mathcal{L}=0$. Thus, if we demand $X u^{i}=1$ for some $u^{i} \in \mathbf{u}$ and $X u^{j}=0$ for any $j \neq i$, we get

$$
\tilde{X}=\frac{\partial}{\partial u^{i}} \Rightarrow \tilde{X} \mathcal{L}=\frac{\partial \mathcal{L}}{\partial u^{i}}=0
$$

This means that $u^{i}$ is a cyclic variable and the dynamics can be reduced. On the other hand, the constant of motion $Q$ becomes

$$
Q=\alpha^{A}(\mathbf{q}) P_{A}=\alpha^{A}(\mathbf{q}) \frac{\partial \mathcal{L}}{\partial \dot{q}^{A}}=\alpha^{A}(\mathbf{q})\left(\frac{\partial \mathcal{L}}{\partial u^{B}} \frac{\partial u^{B}}{\partial \dot{q}^{A}}+\frac{\partial \mathcal{L}}{\partial \dot{u}^{B}} \frac{\partial \dot{u}^{B}}{\partial \dot{q}^{A}}\right) .
$$

Since $\mathbf{q} \rightarrow \mathbf{u}$ is a point transformation, we have $\frac{\partial u^{B}}{\partial \dot{q}^{A}}=0$ and $\frac{\partial \dot{u}^{B}}{\partial \dot{q}^{A}}=\frac{\partial u^{B}}{\partial q^{A}}$, Therefore,

$$
Q=\alpha^{A}(\mathbf{q}) \frac{\partial u^{B}}{\partial q^{A}} \frac{\partial \mathcal{L}}{\partial \dot{u}^{B}}=X u^{B} \frac{\partial \mathcal{L}}{\partial \dot{u}^{B}}=\frac{\partial \mathcal{L}}{\partial \dot{u}^{i}}=\pi_{i},
$$

where $\pi_{i}$ is the momentum conjugate to $u^{i}$. Thus, as expected the constant of motion which corresponds to the Noether symmetry is nothing but the momentum conjugated to the cyclic variable. Now, it is obvious that $\left[\hat{\pi}_{i}, H\right]=0$ and the quantum description of the system under consideration can be given by the following equations

$$
\left\{\begin{array}{l}
\mathcal{H} \Psi(\mathbf{q})=0 \\
\hat{\pi}_{i} \Psi(\mathbf{q})=\sigma_{i} \Psi(\mathbf{q})
\end{array}\right.
$$


where $\sigma_{i}$ is the eigenvalue of $\hat{\pi}_{i}$.

In the next section we will apply the above formalism to a given cosmological model as a dynamical system. In cosmological systems, since the scale factors, matter fields and their conjugate momenta play the role of dynamical variables, introduction of Noether symmetry by adopting the approach discussed above is particularly relevant.

\section{Scalar field cosmology}

In this section we consider a FRW cosmology with a scalar field with which the action of the model is augmented. In a quasi-spherical polar coordinate the geometry of such a space-time is described by the metric

$$
d s^{2}=-N^{2}(t) d t^{2}+a^{2}(t)\left[\frac{d r^{2}}{1-k r^{2}}+r^{2}\left(d \theta^{2}+\sin ^{2} \theta d \phi^{2}\right)\right],
$$

where $N(t)$ is the lapse function, $a(t)$ the scale factor and $k=0, \pm 1$ is the curvature index. Since our goal is to study the models which exhibit Noether symmetry, we do not include any matter contribution in the action. Let us start from the action (we work in units where $c=\hbar=16 \pi G=1$ )

$$
\mathcal{S}=\int d^{4} x \sqrt{-g}\left[R-\frac{1}{2} \epsilon g^{\mu \nu} \partial_{\mu} \phi \partial_{\nu} \phi-V(\phi)\right]
$$

where $g$ is the determinant of the metric tensor $g_{\mu \nu}, R$ is the Ricci scalar corresponding to $g_{\mu \nu}, V(\phi)$ is the potential function for the scalar field $\phi(t)$ and the parameter $\epsilon= \pm 1$ corresponds to the ordinary scalar field (where $\epsilon=+1$ ) or phantom scalar field (where $\epsilon=-1$ ). By substituting (18) into (19) and integrating over spatial dimensions, we are led to a point-like Lagrangian in the minisuperspace $\{a, \phi\}$ as

$$
\mathcal{L}=-3 a \dot{a}^{2}+\frac{1}{2} \epsilon a^{3} \dot{\phi}^{2}+3 k a-a^{3} V(\phi),
$$

in which we have set $N=1$ so that the time parameter $t$ is the usual cosmic time. Now, it is easy to see that this minisuperspace has the following minisuper metric

$$
\mathcal{G}_{A B} d q^{A} d q^{B}=-6 a d a^{2}+\epsilon a^{3} d \phi^{2} .
$$

This two dimensional minisuperspace is the space spanned with the dynamical variables of the model and indeed is the space in which we are looking for a Noether symmetry. In the following we will apply the Noether symmetry approach to the minisuperspace which is represented by a curved manifold with a minisuper metric given by (21).

\subsection{Ordinary scalar field}

In this case $(\epsilon=+1)$ the minisuperspace has a Lorentzian metric. However, a simple calculation shows that its Ricci scalar is zero and therefore this space is a two dimensional Lorentzian flat space. This means that there is a suitable change of variables that can transform the metric into a Minkowskian one. To do this, consider the following change of variables $q^{A}=(a, \phi) \rightarrow Q^{A}=(x, y)[14]$

$$
x=\frac{1}{\omega} a^{3 / 2} \cosh (\omega \phi), \quad y=\frac{1}{\omega} a^{3 / 2} \sinh (\omega \phi),
$$

where $\omega=\sqrt{6} / 4$. In terms of these new variables, Lagrangian (20) takes the form

$$
\mathcal{L}=\frac{1}{2}\left(\dot{x}^{2}-\dot{y}^{2}\right)-\frac{1}{2} \omega^{2}\left(x^{2}-y^{2}\right) V\left(\frac{y}{x}\right)+3 k \omega^{2 / 3}\left(x^{2}-y^{2}\right)^{1 / 3},
$$

with the corresponding Hamiltonian becoming

$$
\mathcal{H}=\frac{1}{2}\left(P_{x}^{2}-P_{y}^{2}\right)+\frac{1}{2} \omega^{2}\left(x^{2}-y^{2}\right) V\left(\frac{y}{x}\right)-3 k \omega^{2 / 3}\left(x^{2}-y^{2}\right)^{1 / 3},
$$


where $P_{x}$ and $P_{y}$ are the momenta conjugate to $x$ and $y$ respectively. Thus, it is easy to see that in the minisuperspace constructed by $Q^{A}=(x, y)$, the metric is Minkowskian and represented by

$$
\overline{\mathcal{G}}_{A B} d Q^{A} d Q^{B}=-d y^{2}+d x^{2} .
$$

Now, we have a set of variables $(x, y)$ endowing the minisuperspace with a Minkowskian metric and we are going to find the potential function $V(y / x)$ such that the dynamical system described with the Lagrangian (23) exhibits the issue of the Noether symmetry. For this purpose, following the steps after equation (8) we define the generator of the desired Noether symmetry as the vector field

$$
X=\alpha \frac{\partial}{\partial x}+\beta \frac{\partial}{\partial y}+\dot{\alpha} \frac{\partial}{\partial \dot{x}}+\dot{\beta} \frac{\partial}{\partial \dot{y}},
$$

on the tangent space of the corresponding configuration space such that the Lie derivative of the Lagrangian with respect to this vector field vanishes, that is, $L_{X} \mathcal{L}=0$. In (26) $\alpha$ and $\beta$ are some functions of $x$ and $y$ which in order to obtain them we use the Noether symmetry condition. For Lagrangian (23) this condition results in

$$
\begin{aligned}
& \alpha\left[-\omega^{2} x V(z)+\frac{1}{2} \omega^{2} \frac{y}{x^{2}}\left(x^{2}-y^{2}\right) V^{\prime}(z)+2 k \omega^{2 / 3} x\left(x^{2}-y^{2}\right)^{-2 / 3}\right]+ \\
& \beta\left[\omega^{2} y V(z)-\frac{1}{2} \omega^{2} \frac{1}{x}\left(x^{2}-y^{2}\right) V^{\prime}(z)-2 k \omega^{2 / 3} y\left(x^{2}-y^{2}\right)^{-2 / 3}\right]+ \\
& \left(\frac{\partial \alpha}{\partial x} \dot{x}+\frac{\partial \alpha}{\partial y} \dot{y}\right) \dot{x}-\left(\frac{\partial \beta}{\partial x} \dot{x}+\frac{\partial \beta}{\partial y} \dot{y}\right) \dot{y}=0,
\end{aligned}
$$

where $z=y / x$ and $V^{\prime}(z)=d V / d z$. Now, we are led to the following system of equations

$$
\begin{gathered}
\omega^{2} V(z)(\beta y-\alpha x)+\frac{1}{2} \omega^{2} V^{\prime}(z)\left(x^{2}-y^{2}\right)\left(\alpha \frac{y}{x^{2}}-\beta \frac{1}{x}\right)+2 k \omega^{2 / 3}\left(x^{2}-y^{2}\right)^{-2 / 3}(\alpha x-\beta y)=0 \\
\frac{\partial \alpha}{\partial x}=\frac{\partial \beta}{\partial y}=0, \quad \frac{\partial \alpha}{\partial y}-\frac{\partial \beta}{\partial x}=0 .
\end{gathered}
$$

Equations (29) admit the independent solutions as

$$
(\alpha, \beta)=(1,0), \quad(\alpha, \beta)=(0,1), \quad(\alpha, \beta)=(y, x),
$$

which upon substitution into relation (28) yields the potential function. In what follows we restrict ourselves to the flat case $k=0$ and consider three cases separately.

3.1.1 The case: $(\alpha, \beta)=(1,0)$

This solution is related to the Noether symmetry generator $X=\frac{\partial}{\partial x}$ and Noether conserved charge $Q=P_{x}$. In this case from equation (28) we obtain

$$
V\left(\frac{y}{x}\right)=\frac{y^{2}}{y^{2}-x^{2}}
$$

In terms of the old variables $(a, \phi)$ this potential is $V(\phi) \sim \sinh ^{2}(\omega \phi)$. With the choose of this potential the Hamiltonian of the model becomes

$$
\mathcal{H}=\frac{1}{2}\left(P_{x}^{2}-P_{y}^{2}\right)-\frac{1}{2} \omega^{2} y^{2} .
$$

This Hamiltonian is nothing but the difference of a free particle and a harmonic oscillator Hamiltonians. Classically, this means that the motion in $y$-direction is bounded and quantum mechanically 
it becomes natural to require the corresponding wave function to be normalizable. Now, the classical and quantum solutions of the model described by Hamiltonian (32) can be easily obtained. The classical dynamics is governed by the Hamiltonian equations, that is

$$
\left\{\begin{array}{l}
\dot{x}=\{x, \mathcal{H}\}=P_{x}, \quad \dot{P}_{x}=\left\{P_{x}, \mathcal{H}\right\}=0, \\
\dot{y}=\{y, \mathcal{H}\}=-P_{y}, \quad \dot{P}_{y}=\left\{P_{y}, \mathcal{H}\right\}=\omega^{2} y,
\end{array}\right.
$$

These equations can be immediately integrated to yield

$$
x(t)=P_{0 x} t+x_{0}, \quad P_{x}=P_{0 x},
$$

and

$$
y(t)=\frac{P_{0 x}}{\omega} \sin \left(\omega t+\delta_{0}\right), \quad P_{y}(t)=-P_{0 x} \cos \left(\omega t+\delta_{0}\right),
$$

where $P_{0 x}, x_{0}$ and $\delta_{0}$ are integration constants. Since in the quantum version of the model we are interested in constructing wave packets from the WDW equation, we would like to obtain a classical trajectory in configuration space $(x, y)$, where the classical time $t$ is eliminated. This is because no such parameter exists in the WDW equation. It is easy to see that the classical solutions (34) and (35) may be displayed as the following trajectories

$$
y=\frac{P_{0 x}}{\omega} \sin \left[\frac{\omega}{P_{0 x}}\left(x-x_{0}\right)+\delta_{0}\right] .
$$

Going back to the old variables $(a, \phi)$ we get the cosmological solutions from (22) as

$$
a(t)=\omega^{2 / 3}\left[\left(P_{0 x} t+x_{0}\right)^{2}-\frac{P_{0 x}^{2}}{\omega^{2}} \sin ^{2}\left(\omega t+\delta_{0}\right)\right]^{1 / 3},
$$

and

$$
\phi(t)=\frac{1}{\omega} \operatorname{Arctanh}\left[\frac{\frac{P_{0 x}}{\omega} \sin \left(\omega t+\delta_{0}\right)}{P_{0 x} t+x_{0}}\right] .
$$

We now focus attention on the study of the quantization of the model described above. This can be achieved via canonical quantization procedure which leads the WDW equation. For the Hamiltonian (32) this equation reads

$$
\left(-\frac{\partial^{2}}{\partial x^{2}}+\frac{\partial^{2}}{\partial y^{2}}-\omega^{2} y^{2}\right) \Psi(x, y)=0 .
$$

We separate the variables in this equation as $\Psi(x, y)=e^{i \nu x} \mathcal{Y}(y)$ leading to $\frac{d^{2} \mathcal{Y}}{d y^{2}}+\left(\nu^{2}-\omega^{2} y^{2}\right) \mathcal{Y}(y)=0$, where $\nu$ is a separation constant. The physical acceptable solutions to this equation can be found in terms of the Hermite polynomials $H_{n}(z)$, if $\nu^{2}=(2 n+1) \omega=\nu_{n}^{2} \omega$, for some integer $n$. Thus, the eigenfunctions of the WDW equation can be written as

$$
\Psi_{n}(X, Y)=e^{i \nu_{n} X} e^{-\frac{1}{2} Y^{2}} H_{n}(Y),
$$

where $(X, Y)=\sqrt{\omega}(x, y)$. There still remains the question of the boundary conditions on the solutions to the WDW equation. Note that the minisuperspace of the above model is a two dimensional manifold $0<a<\infty,-\infty<\phi<+\infty$. According to [15], its nonsingular boundary is the line $a=0$ with $|\phi|<\infty$, while at the singular boundary, at least one of the two variables is infinite. In terms of the variables $x$ and $y$, introduced in (22), the minisuperspace is recovered by $x>0, x>|y|$, and the nonsingular boundary may be represented by $x=y=0$. Since the minisuperspace variables are restricted to the above mentioned domain, the minisuperspace quantization deals only with wave functions defined on this region. Therefore, to construct the quantum version of the model one should 
take into account this issue. This is because that in such cases one usually has to impose boundary conditions on the allowed wave functions otherwise the relevant operators, specially the Hamiltonian, will not be self-adjoint. The condition for the Hamiltonian operator $\hat{\mathcal{H}}$ associated with the classical Hamiltonian function $(24)$ to be self-adjoint is $\left(\psi_{1}, \hat{\mathcal{H}} \psi_{2}\right)=\left(\hat{\mathcal{H}} \psi_{1}, \psi_{2}\right)$ or

$$
\int_{\Omega} \psi_{1}^{*}(x, y) \hat{\mathcal{H}} \psi_{2}(x, y) d x d y=\int_{\Omega} \psi_{2}(x, y) \hat{\mathcal{H}} \psi_{1}^{*}(x, y) d x d y,
$$

where the integrals should be taken over the domain where the minisuperspace variables are defined on which. Following the calculations in [16] and dealing only with square integrable wave functions, this condition yields a vanishing wave function at nonsingular boundary of the minisuperspace. Hence, we impose the boundary condition on the solutions (40) such that at the nonsingular boundary (at $a=0$ and $|\phi|<\infty)$ the wave function vanishes. This makes the Hamiltonian hermitian and self-adjoint and can avoid the singularities of the classical theory, i.e. there is zero probability for observing a singularity corresponding to $a=0$. Therefore, we require

$$
\Psi(a=0, \phi)=0 \Rightarrow \Psi(X=0, Y=0)=0,
$$

which yields

$$
H_{n}(0)=0 \Rightarrow n=\text { odd }
$$

In general, one of the most important features in quantum cosmology is the recovery of classical cosmology from the corresponding quantum model or, in other words, how can the WDW wave functions predict a classical universe. In this approach, one usually constructs a coherent wave packet with good asymptotic behavior in the minisuperspace, peaking in the vicinity of the classical trajectory [17]. Therefore, we may now write the general solutions to the WDW equation as a superposition of the eigenfunctions, that is

$$
\Psi(X, Y)=\sum_{n=0}^{\infty} c_{n} e^{i \nu_{n} X} e^{-\frac{1}{2} Y^{2}} H_{2 n+1}(Y)
$$

where $c_{n}$ are suitable weight factors to construct the wave packets and to make a convergent superposition, we may choose them such that the summands with smaller $n$ have more important contribution to the above superposition. Therefore, to achieve an analytical expression for the wave function we assume that the above superposition is taken over such values of $n$ for which one can use the approximation $\nu_{n} \sim 2 n+1$, so that

$$
\Psi(X, Y)=\sum_{n=0}^{\infty} c_{n} e^{i(2 n+1) X} e^{-\frac{1}{2} Y^{2}} H_{2 n+1}(Y) .
$$

By using the equality

$$
\sum_{n=0}^{\infty} \frac{\gamma^{2 n+1}}{(2 n+1) !} H_{2 n+1}(z)=e^{-\gamma^{2}} \sinh 2 \gamma z,
$$

we can evaluate the sum over $n$ in (45) and simple analytical expression for this sum is found if we choose $\gamma$ to be $e^{i X}$ and $c_{n}=\mathcal{N} /(2 n+1)$ !, which results in $^{1}$

$$
\Psi(X, Y)=\mathcal{N} e^{-\frac{1}{2} Y^{2}} \exp \left(-e^{2 i X}\right) \sinh \left(2 Y e^{i X}\right)
$$

where $\mathcal{N}$ is a numerical factor. Before going any further, some remarks are in order. An important ingredient in any model theory related to the quantization of a cosmological setting is the choice

\footnotetext{
${ }^{1}$ One may have some doubts on this final form for the wave function and the following results due to the assumption $\nu_{n} \sim 2 n+1$ which seems to be irrelevant especially in the case of a discrete spectrum for $n$. To overcome this problem, we have made a numerical study of the behavior of $|\Psi|^{2}$ based on equation (44) with $c_{n}=1 /(2 n+1)$ ! and $\nu_{n}=(2 n+1)^{2}$ and have verified that the general patterns of the resulting wave packets follow the behavior shown in figure 1 with a very good approximation.
} 

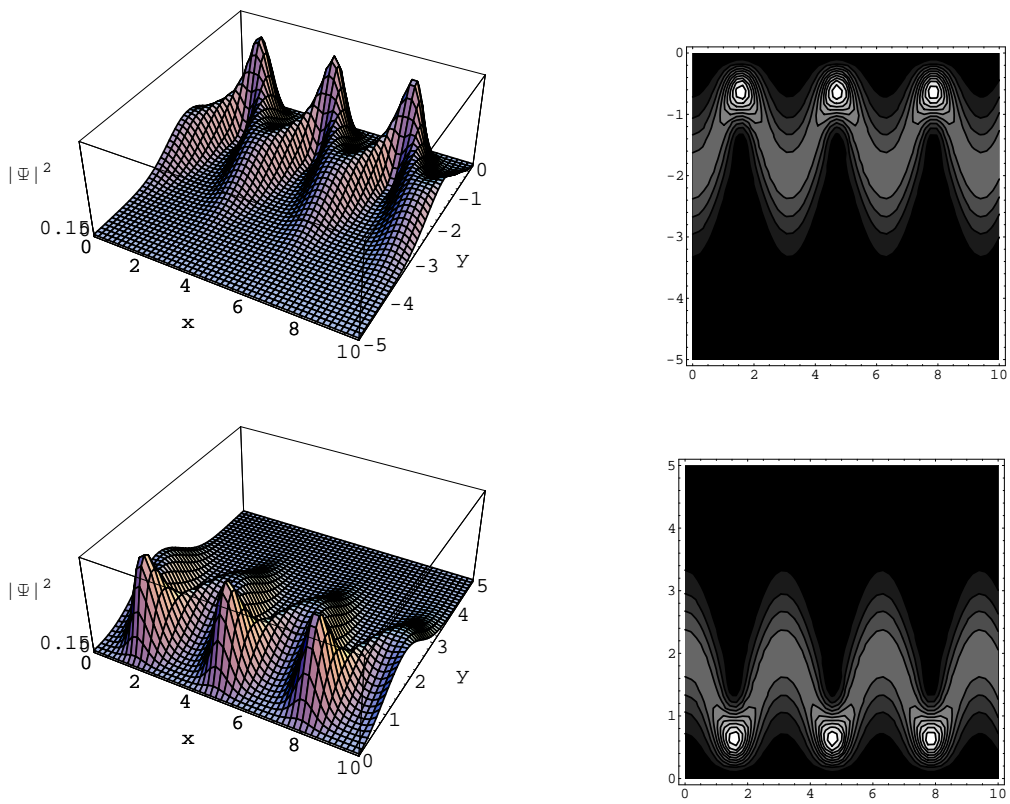

Figure 1: The figures show $|\Psi(X, Y)|^{2}$, the square of the wave function and its corresponding contour plot. Up: the figures are plotted for negative values of $y$ and bottom: the same figures are plotted for positive values of $y$.

of a probability measure in order to make predictions from a given solution to the WDW equation. Since the WDW equation is a second order Klaein-Gordon type equation in quantum field theory, the most widely used probability density for it results the conserved current $j=\frac{i}{2}\left(\Psi^{*} \nabla \Psi-\Psi \nabla \Psi^{*}\right)$. But this immediately presents a problem, because the probability density given by this expression is not positive definite. For this reason and some other difficulties related to this interpretation, an alternative used approach is to introduce $|\Psi|^{2}$ as the probability density. Although this definition is necessarily positive definite and specially works very well for the minisuperspace models, it suffers from some problems. The most important of such problems arise when we are dealing with the non-normalizable. Despite of the existence of the above mentioned problems, here we shall look for the peaks in the wave function in the sense that if a peak is sufficiently strong in some region, the probability of the universe being in this region is large. In figure 1 we have plotted the square of the wave function (47) and its corresponding contour plot. It is seen that the peaks of the wave function follow a periodic pattern. In comparison with the classical path (36), this means that a good correlation exists between the quantum patterns shown in this figure and the classical trajectory (36) in configuration space $(x, y)$.

\subsubsection{The case: $(\alpha, \beta)=(0,1)$}

This case relates the Noether vector $\frac{\partial}{\partial y}$ and its corresponding conserved charge is $Q=P_{y}$. From (28) the potential function reads as

$$
V\left(\frac{y}{x}\right)=\frac{x^{2}}{x^{2}-y^{2}}
$$

where in terms of the old variables $(a, \phi)$ can be termed as $V(\phi) \sim \cosh ^{2}(\omega \phi)$. Since the Hamiltonian of the model, in this case is

$$
\mathcal{H}=\frac{1}{2}\left(P_{x}^{2}-P_{y}^{2}\right)+\frac{1}{2} \omega^{2} x^{2},
$$

we see that there is no major difference between this case the the previous one except that the variables $x$ and $y$ change their role with each other. Therefore, following the steps (32)-(47), we are 
led to the classical solutions

$$
\begin{gathered}
x(t)=\frac{P_{0 y}}{\omega} \sin \left(\omega t+\eta_{0}\right), \quad P_{x}=P_{0 y} \cos \left(\omega t+\eta_{0}\right), \\
y(t)=-P_{0 y} t+y_{0}, \quad P_{y}(t)=P_{0 y},
\end{gathered}
$$

and the corresponding classical trajectories

$$
x=\frac{P_{0 y}}{\omega} \sin \left[\frac{\omega}{P_{0 y}}\left(y_{0}-y\right)+\eta_{0}\right],
$$

where in terms of the old variables $(a, \phi)$ can be viewed as

$$
a(t)=\omega^{2 / 3}\left[\frac{P_{0 y}^{2}}{\omega^{2}} \sin ^{2}\left(\omega t+\eta_{0}\right)-\left(-P_{0 y} t+y_{0}\right)^{2}\right]^{1 / 3},
$$

and

$$
\phi(t)=\frac{1}{\omega} \operatorname{Arctanh}\left[\frac{-P_{0 y} t+y_{0}}{\frac{P_{0 y}}{\omega} \sin \left(\omega t+\eta_{0}\right)}\right] .
$$

Also, the wave function of the quantum counterpart of the model can be written as

$$
\Psi(X, Y)=\mathcal{N} e^{-\frac{1}{2} X^{2}} \exp \left(-e^{2 i Y}\right) \sinh \left(2 X e^{i Y}\right) .
$$

The discussions on the comparison between quantum cosmological solution and its classical version are the same as previous model, namely the $(\alpha, \beta)=(1,0)$ model. Similar discussion as above would be applicable to this case as well.

\subsubsection{The case: $(\alpha, \beta)=(y, x)$}

In this case the Noether symmetry is generated by the following vector field

$$
X=y \frac{\partial}{\partial x}+x \frac{\partial}{\partial y}+\dot{y} \frac{\partial}{\partial \dot{x}}+\dot{x} \frac{\partial}{\partial \dot{y}},
$$

which corresponds to the Noether conserved charged $Q=y P_{x}+x P_{y}$. The condition (28), in this case, demands that the potential function be a constant function and as such, we choose it to be 1 . Therefore, the Hamiltonian takes the form

$$
\mathcal{H}=\frac{1}{2}\left(P_{x}^{2}-P_{y}^{2}\right)+\frac{1}{2} \omega^{2}\left(x^{2}-y^{2}\right)
$$

which describes an isotropic oscillator-ghost-oscillator system. The classical equations of motion are given by

$$
\left\{\begin{array}{c}
\dot{x}=\{x, \mathcal{H}\}=P_{x}, \quad \dot{P}_{x}=\left\{P_{x}, \mathcal{H}\right\}=-\omega^{2} x \\
\dot{y}=\{y, \mathcal{H}\}=-P_{y}, \quad \dot{P}_{y}=\left\{P_{y}, \mathcal{H}\right\}=\omega^{2} y
\end{array}\right.
$$

Choosing the integration constants such that the solutions satisfy the zero energy condition $\mathcal{H}=0$, the solutions are obtained as

$$
\begin{cases}x(t)=A \sin \left(\omega t+\eta_{0}\right), & P_{x}(t)=A \omega \cos \left(\omega t+\eta_{0}\right), \\ y(t)=\ell A \sin \left(\omega t+\delta_{0}\right) & P_{y}(t)=-\ell A \omega \cos \left(\omega t+\delta_{0}\right),\end{cases}
$$


where $A, \delta_{0}$ and $\eta_{0}$ are integration constants and $\ell= \pm 1$. From the above equations, we see that the classical trajectories obey the relation

$$
y^{2}+x^{2}-2 \ell x y \cos \left(\delta_{0}-\eta_{0}\right)-A^{2} \sin ^{2}\left(\delta_{0}-\eta_{0}\right)=0 .
$$

This equation describes ellipses which their major axes make angle $\pi / 4$ with the positive/negative $x$-axis according to the choices \pm 1 for $\ell$. Also, the eccentricity and the size of each trajectory are determined by $\left(\delta_{0}-\eta_{0}\right)$ and $A$ respectively. Now, using the transformation (22) the classical cosmological behavior can be obtained as

$$
a(t)=A^{2 / 3} \omega^{2 / 3} \sin ^{1 / 3}\left(\delta_{0}-\eta_{0}\right) \sin ^{1 / 3}\left(2 \omega t+\delta_{0}+\eta_{0}\right),
$$

and

$$
\phi(t)=\frac{1}{\omega} \operatorname{Arctanh}\left[\frac{\ell \sin \left(\omega t+\delta_{0}\right)}{\sin \left(\omega t+\eta_{0}\right)}\right] .
$$

At this step, as the previous subsections, we deal with the quantization of the model. The WDW equation corresponding to the Hamiltonian (57) reads

$$
\left(-\frac{\partial^{2}}{\partial x^{2}}+\frac{\partial^{2}}{\partial y^{2}}+\omega^{2} x^{2}-\omega^{2} y^{2}\right) \Psi(x, y)=0
$$

This equation is a quantum isotropic oscillator-ghost-oscillator system with zero energy. Therefore, its solutions belong to a subspace of the Hilbert space spanned by separable eigenfunctions of a two-dimensional isotropic simple harmonic oscillator Hamiltonian. In [18] the models of coupled harmonic oscillators have been studied in other contexts relevant for quantum cosmology. Separating the eigenfunctions of (63) in the form

$$
\Psi_{n_{1}, n_{2}}(X, Y)=\mathcal{X}_{n_{1}}(X) \mathcal{Y}_{n_{2}}(Y)
$$

yields

$$
\mathcal{X}_{n_{1}}(X)=e^{-\frac{1}{2} X^{2}} H_{n_{1}}(X), \quad \mathcal{Y}_{n_{2}}(Y)=e^{-\frac{1}{2} Y^{2}} H_{n_{2}}(Y),
$$

subject to the restriction $n_{1}=n_{2}=n$. In (65), $H_{n}(z)$ are the Hermite polynomials and $(X, Y)=$ $\sqrt{\omega}(x, y)$ as before. Applying again the boundary condition (42), we are led to the following general solution to the WDW equation

$$
\Psi(X, Y)=\sum_{n_{1}=0}^{\infty} \sum_{n_{2}=0}^{\infty} c_{n_{1}} c_{n_{2}} e^{-\frac{1}{2}\left(X^{2}+Y^{2}\right)} H_{2 n_{1}+1}(X) H_{2 n_{2}+1}(Y) .
$$

If we choose the coefficients $c_{n_{1}}$ and $c_{n_{2}}$ to be $c_{n_{1}}=\xi^{2 n_{1}+1} /\left(2 n_{1}+1\right)$ ! and $c_{n_{2}}=\zeta^{2 n_{2}+1} /\left(2 n_{2}+1\right)$ ! where $\xi$ and $\zeta$ are arbitrary complex constants, the wave function takes the form

$$
\Psi(X, Y)=\mathcal{N} e^{-\frac{1}{2}\left(X^{2}+Y^{2}\right)} e^{-\left(\xi^{2}+\zeta^{2}\right)} \sinh (2 \xi X) \sinh (2 \zeta Y) .
$$

In figure 2, we have shown the qualitative behavior of this wave function and its contour plot. To realize the correlation between these quantum patterns and the classical trajectories represented by (60), note that in the minisuperspace formulation, the cosmic evolution of the universe is modeled with the motion of a point particle in a space with minisuperspace coordinates. In motion on an ellipse, the particle (universe) spend most of its time in the region near its apogees. This means that the particle will be found around the apogees of its trajectory with the maximum probability. This is just what the figure 2 is showing. As it is seen from this figure, the wave function has two pair peaks, each pair may be interpreted as two apogees of the classical trajectory. In classical model, the transition from one point on the configuration space to any other points will be described by a continuous motion on the classical trajectory. The quantum description of such a transition, on the other hand, may be explained by a tunneling procedure. This means that there are different possible states from which our present universe could have evolved and tunneled in the past, from one state to another. Again, we see that our quantization leads us to a model free of the classical singularity and a good correlation with its classical counterpart. 

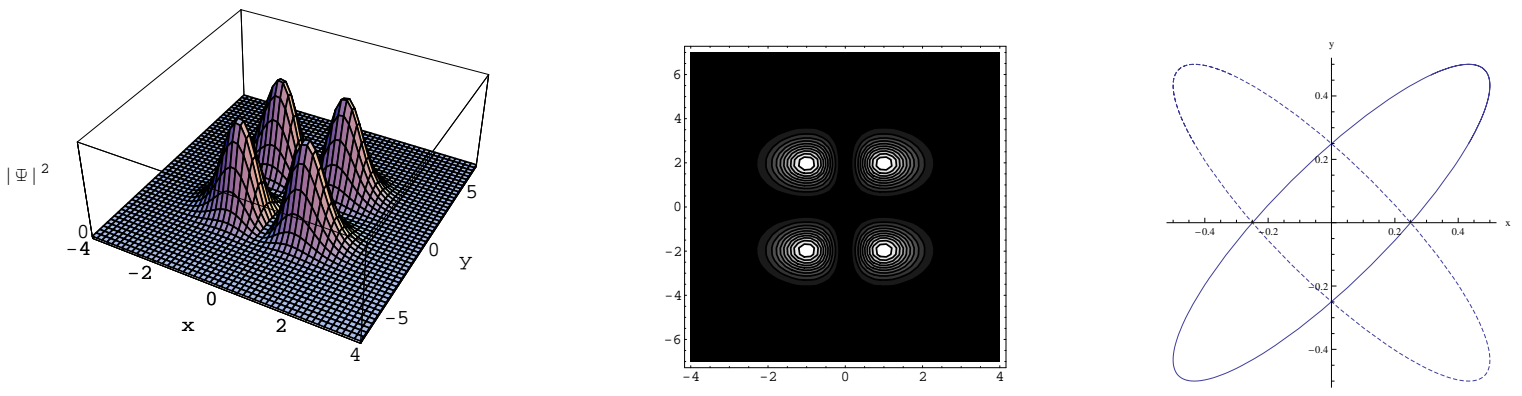

Figure 2: The figures show $|\Psi(X, Y)|^{2}$, the square of the wave function (67), its corresponding contour plot and the classical trajectory (60), (solid line for $\ell=1$ and dashed line for $\ell=-1$ ).

\subsection{Phantom scalar field}

In this case $(\epsilon=-1)$, the minisuperspace manifold would have an Euclidean signature (see $(21)$ ) with zero Ricci scalar. To write the metric in its canonical form, we apply the transformation

$$
x=\frac{1}{\omega} a^{3 / 2} \cos (\omega \phi), \quad y=\frac{1}{\omega} a^{3 / 2} \sin (\omega \phi) .
$$

It is easy to see that in terms of these new variables the metric takes the form of a 2-dimensional Euclidean one represented by

$$
\overline{\mathcal{G}}_{A B} d Q^{A} d Q^{B}=-d x^{2}-d y^{2} .
$$

Also, the phantom counterpart of Lagrangian (20) becomes (we consider $k=0$ )

$$
\mathcal{L}=\frac{1}{2}\left(-\dot{x}^{2}-\dot{y}^{2}\right)-\frac{1}{2} \omega^{2}\left(x^{2}+y^{2}\right) V\left(\frac{y}{x}\right)
$$

with the corresponding Hamiltonian becoming

$$
\mathcal{H}=\frac{1}{2}\left(-P_{x}^{2}-P_{y}^{2}\right)+\frac{1}{2} \omega^{2}\left(x^{2}+y^{2}\right) V\left(\frac{y}{x}\right) .
$$

Again, considering a Noether symmetry generated by a vector field like (26), the condition $L_{X} \mathcal{L}=0$ will lead us to the following equations for $\alpha$ and $\beta$

$$
\begin{gathered}
-\omega^{2} V(z)(\beta y+\alpha x)+\frac{1}{2} \omega^{2} V^{\prime}(z)\left(x^{2}+y^{2}\right)\left(\alpha \frac{y}{x^{2}}-\beta \frac{1}{x}\right)=0, \\
\frac{\partial \alpha}{\partial x}=\frac{\partial \beta}{\partial y}=0, \quad \frac{\partial \alpha}{\partial y}+\frac{\partial \beta}{\partial x}=0,
\end{gathered}
$$

where $z=y / x$ and $V^{\prime}(z)=d V / d z$ as before. The independent solutions of the system (73) my be obtained as

$$
(\alpha, \beta)=(1,0), \quad(\alpha, \beta)=(0,1), \quad(\alpha, \beta)=(y,-x) .
$$

Now, following the same procedure as in the previous subsection, we consider the above tree case separately to study their corresponding classical and quantum cosmology.

\subsubsection{The case: $(\alpha, \beta)=(1,0)$}

This case corresponds to the vector field $X=\frac{\partial}{\partial x}$ which results the conserved quantity $Q=P_{x}$. The potential function can be obtained from (72) as 


$$
V\left(\frac{y}{x}\right)=\frac{y^{2}}{x^{2}+y^{2}}
$$

where in terms of the cosmic variables $(a, \phi)$ may be written as $V(\phi) \sim \sin ^{2}(\omega \phi)$. This potential function transforms the Hamiltonian (71) into the form

$$
\mathcal{H}=\frac{1}{2}\left(-P_{x}^{2}-P_{y}^{2}\right)+\frac{1}{2} \omega^{2} y^{2},
$$

from which one gets the following classical equations of motion

$$
\left\{\begin{array}{l}
\dot{x}=\{x, \mathcal{H}\}=-P_{x}, \quad \dot{P}_{x}=\left\{P_{x}, \mathcal{H}\right\}=0, \\
\dot{y}=\{y, \mathcal{H}\}=-P_{y}, \quad \dot{P}_{y}=\left\{P_{y}, \mathcal{H}\right\}=-\omega^{2} y,
\end{array}\right.
$$

with solutions

$$
x(t)=-P_{0 x} t+x_{0}, \quad P_{x}=P_{0 x},
$$

and

$$
y(t)=\frac{P_{0 x}}{\omega} \cosh \left(\omega t+\delta_{0}\right), \quad P_{y}(t)=-P_{0 x} \sinh \left(\omega t+\delta_{0}\right),
$$

where for the sake of the simplicity, we use the same notation as in the previous section for the integration constants. These solutions obey the classical trajectories in the configuration space $(x, y)$

$$
y=\frac{P_{0 x}}{\omega} \cosh \left(\frac{\omega}{P_{0 x}}\left(x_{0}-x\right)+\delta_{0}\right) .
$$

Finally, going back to the original variables $(a, \phi)$, the corresponding classical cosmology will be obtained as

$$
a(t)=\omega^{2 / 3}\left[\left(-P_{0 x} t+x_{0}\right)^{2}+\frac{P_{0 x}^{2}}{\omega^{2}} \cosh ^{2}\left(\omega t+\delta_{0}\right)\right]^{1 / 3},
$$

and

$$
\phi(t)=\frac{1}{\omega} \operatorname{Arctan}\left[\frac{\frac{P_{0 x}}{\omega} \cosh \left(\omega t+\delta_{0}\right)}{-P_{0 x} t+x_{0}}\right] .
$$

We see that unlike the case of the ordinary scalar field, here all the motions are unbounded, so that it becomes natural to expect that the wave function describing the quantum version of the model to be non-normalizable. Now, by canonical quantization the WDW equation is given by

$$
\left(\frac{\partial^{2}}{\partial x^{2}}+\frac{\partial^{2}}{\partial y^{2}}+\omega^{2} y^{2}\right) \Psi(x, y)=0
$$

The eigenfunctions of this equation can be found by the method of separation of variables, by means of which we obtain

$$
\Psi_{\nu}(X, Y)=e^{ \pm i \nu X} \mathcal{D}_{-\frac{1}{2} \pm i \frac{\nu^{2}}{2}}(( \pm 1+i) Y),
$$

where $\nu$ is the separation constant and $\mathcal{D}$ is the parabolic cylinder function. As before the general solutions may be constructed by a superposition of these eigenfunctions. Since non-normalizable states in this case belong to the continuous spectrum, the general wave function should be constructed by a continuous superposition in the form

$$
\Psi(X, Y)=\int_{-\infty}^{+\infty} C(\nu) \Psi_{\nu}(X, Y) d \nu
$$

However, to achieve a more clear picture of the wave function by the numerical methods which would be comparable with the results in the previous section, we restrict ourselves to a discrete superposition 

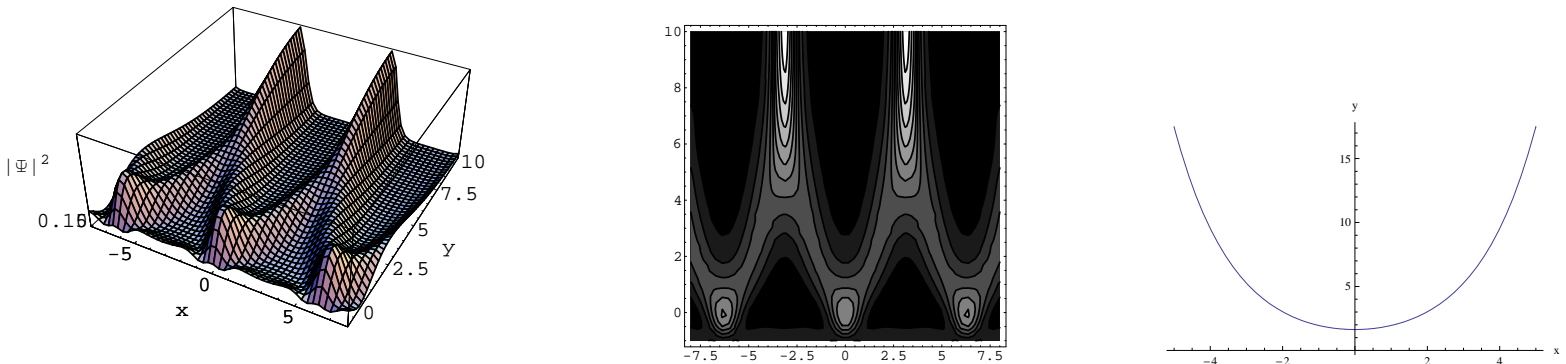

Figure 3: From left to right: $|\Psi(X, Y)|^{2}$, the square of the phantom wave function (86), in which we have taken the combinations from six terms (taking more terms would only have minor effects on the shape of the wave function) with $c_{n}=1 /\left(n ! 2^{n}\right)$, its corresponding contour plot and the classical trajectory $(80)$.

of the above eigenfunctions. Thus, by choosing the separation constant as $\nu^{2}=(2 n-i) \omega=\nu_{n}^{2} \omega$ and connecting the parabolic cylinder function with the Hermite functions [19], the general solution to the WDW equation takes the form

$$
\Psi(X, Y)=\sum_{n=0}^{\infty} c_{n} e^{ \pm i \nu_{n} X} e^{-\frac{1}{2} i Y^{2}} H_{i n}\left(\frac{1}{\sqrt{2}}(1-i) Y\right) .
$$

We have summarized the above results in figure 3. As this figure shows, like in the case of the ordinary scalar field, the evolution of the universe based on the classical cosmology represents a late time expansion. This is because the particle (universe) moves on one of the branches of its classical trajectory forever without any turning points. A remarkable point about this motion is that in spite of the ordinary scalar field case, it does not come from a big-bang singularity in which the scale factor goes to zero, but instead tends to another kind of singularity in which the scale factor diverges. In the models where the phantom fields are considered by a perfect fluid with the equation of state parameter less than -1 , this kind of singularity will be achieved in a finite-time and is called the big-rip singularity. On the other hand a glance at the quantum patterns shows that although a good correlation exists between them and the classical loci in the configuration space, but the quantum effects dominate in the region of the classical singularity, i.e., at the large values of scale factor. In this regime the quantum solutions fall from an expansion phase to a contraction era and this phenomenon will be repeated cyclic. By such a behavior we see that the quantum effects show their important role for the large values of the scale factor and thus the evolution of the scale factor towards a big-rip like singularity will be avoided [20]. However, a remark about the above analysis is that it happens in some internal time parameter such as $x$ and in order to see whether it is physically meaningful the results should translate in terms of the cosmic time $t$. The answer to this question in general may be not an easy task. This is because one usually uses non-standard parametrization of the metric in order to simplify the calculations and have a manageable Lagrangian. Therefore, returning the results into the proper time gauge is not always an integrable process. By the way, in our simple model at hand, since (78) represents a monotonic relation between $x$ and $t$, we may argue that the above conclusions are also valid in terms of the cosmic time gauge $t$ as well.

\subsubsection{The case: $(\alpha, \beta)=(0,1)$}

The situation in this case is alike the one that was occurred when we were discussing about the ordinary scalar field. The potential function resulting from this solution is $V(y / x)=x^{2} /\left(x^{2}+y^{2}\right)$, or $V(\phi) \sim \cos ^{2}(\omega \phi)$ in terms of the variables $(a, \phi)$. Again, all of the results coming from this potential function can be obtained from their counterparts in the previous subsection with exchange the role of the variables $x$ and $y$ with each other and because of this we do not repeat the calculations ones again. 


\subsubsection{The case: $(\alpha, \beta)=(y,-x)$}

This solution generates the Noether symmetry with the vector field

$$
X=y \frac{\partial}{\partial x}-x \frac{\partial}{\partial y}+\dot{y} \frac{\partial}{\partial \dot{x}}-\dot{x} \frac{\partial}{\partial \dot{y}},
$$

which gives the conserved charge $Q=y P_{x}-x P_{y}$. Substitution of this solution into the equation (72) we get a constant value for the potential function and as before we take it to be equal to 1. Therefore, the corresponding dynamical system can be described by the Hamiltonian

$$
\mathcal{H}=\frac{1}{2}\left(-P_{x}^{2}-P_{y}^{2}\right)+\frac{1}{2} \omega^{2}\left(x^{2}+y^{2}\right) .
$$

Following the same steps as in the above sections, we arrive the classical equations of motion as

$$
\begin{cases}\dot{x}=\{x, \mathcal{H}\}=-P_{x}, & \dot{P}_{x}=\left\{P_{x}, \mathcal{H}\right\}=-\omega^{2} x, \\ \dot{y}=\{y, \mathcal{H}\}=-P_{y}, & \dot{P}_{y}=\left\{P_{y}, \mathcal{H}\right\}=-\omega^{2} y\end{cases}
$$

which admit the following integrals

$$
\begin{cases}x(t)=A \cosh \left(\omega t+\eta_{0}\right), & P_{x}(t)=-A \omega \sinh \left(\omega t+\eta_{0}\right), \\ y(t)=\ell A \sinh \left(\omega t+\delta_{0}\right) & P_{y}(t)=-\ell A \omega \cosh \left(\omega t+\delta_{0}\right),\end{cases}
$$

where as before we take $A, \delta_{0}$ and $\eta_{0}$ as integration constants and $\ell= \pm 1$. Now one can eliminate the time parameter $t$ from these solutions to get the classical trajectories in the configuration space $(x, y)$ as

$$
y^{2}-x^{2}-2 \ell x y \sinh \left(\delta_{0}-\eta_{0}\right)+A^{2} \cosh ^{2}\left(\delta_{0}-\eta_{0}\right)=0,
$$

which represents a hyperbola so that the the particle (universe) has an unbounded motion in the $x-y$ plane. Now, going back to the original variables $(a, \phi)$ with the help of the transformation (68), the dynamics of the system reads as

$$
a(t)=A^{2 / 3} \omega^{2 / 3} \cosh ^{1 / 3}\left(\delta_{0}-\eta_{0}\right) \cosh ^{1 / 3}\left(2 \omega t+\delta_{0}+\eta_{0}\right),
$$

and

$$
\phi(t)=\frac{1}{\omega} \operatorname{Arctan}\left[\frac{\ell \sinh \left(\omega t+\delta_{0}\right)}{\cosh \left(\omega t+\eta_{0}\right)}\right] .
$$

To proceed, let us to deal with the quantum cosmology associated with the model described by the Hamiltonian (88). The corresponding WDW equation is

$$
\left(\frac{\partial^{2}}{\partial x^{2}}+\frac{\partial^{2}}{\partial y^{2}}+\omega^{2} x^{2}+\omega^{2} y^{2}\right) \Psi(x, y)=0 .
$$

With the same techniques as in the previous sections and also with same notations one may represent the general solutions to the above equation as

$$
\begin{array}{r}
\Psi(X, Y)=\sum_{n=0}^{\infty} c_{n} e^{-\frac{1}{2} i\left(X^{2}+Y^{2}\right)}\left[H_{i n}\left(\frac{1}{\sqrt{2}}(1-i) X\right) H_{-1-i n}\left(\frac{1}{\sqrt{2}}(1-i) Y\right)+\right. \\
\left.H_{i n}\left(\frac{1}{\sqrt{2}}(1-i) Y\right) H_{-1-i n}\left(\frac{1}{\sqrt{2}}(1-i) X\right)\right] .
\end{array}
$$



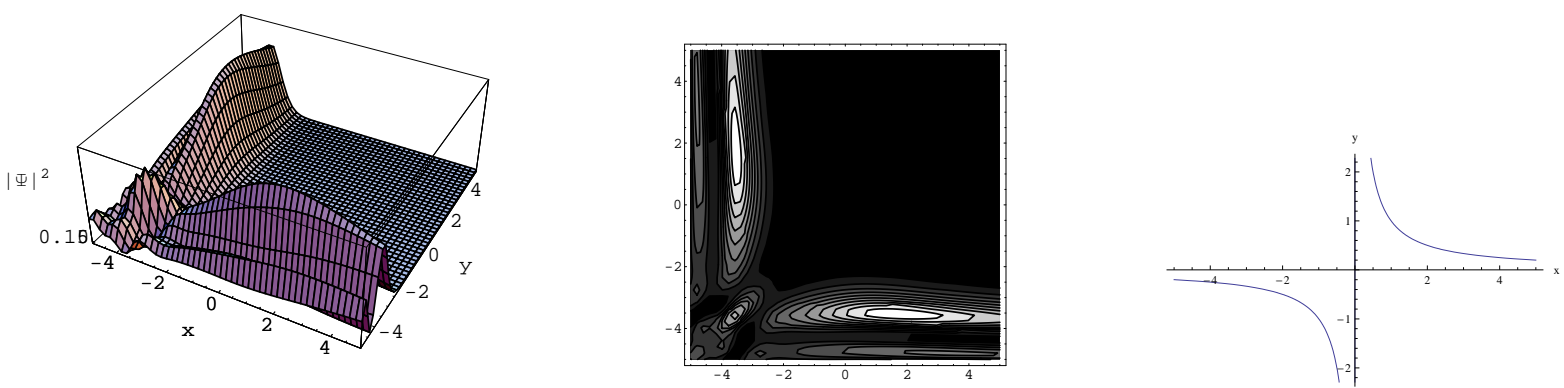

Figure 4: From left to right: $|\Psi(X, Y)|^{2}$, the square of the phantom wave function (95), in which we have taken the combinations from six terms (taking more terms would only have minor effects on the shape of the wave function) with $c_{n}=1 /\left(n ! 2^{n}\right)$, its corresponding contour plot and the classical trajectory (91) with $\ell=1$.

Figure 4 shows the qualitative behavior of the wave function and classical trajectory for typical values of the parameters. As we have mentioned above, the motion in configuration space is an unbounded motion along one of the branches of a hyperbola. A point (universe) with such a motion spend lots of its time far from the apex of its path. This is exactly what we realize from the quantum wave function in figure 4 . These patterns show that the peaks of the wave function follow the classical trajectory with a good degree of accuracy.

\section{Conclusions}

In this paper we have studied the scalar field classical and quantum cosmology in a Noether symmetry point of view. The minisuperspace of such a model is constructed of a two dimensional manifold with vanishing Ricci scalar. Therefore, it is possible to find a coordinate transformation which cast the corresponding metric to a Minkowskian or Euclidean one according to the choices of an ordinary or phantom model for the scalar field. Here, after a brief review of the issue of the Noether symmetry in a dynamical system we have applied the formalism into a FRW cosmological model with a scalar field as its source. The phase space was then constructed by taking the scale factor $a(t)$ and scalar field $\phi(t)$ as the independent dynamical variables. The Lagrangian of the model in the configuration space spanned by $\{a, \phi\}$ is so constructed such that its variation with respect to these dynamical variables yields the appropriate field equations. As is mentioned above, we introduced a new set of variables $(x, y)$ in terms of which the minisuperspace takes the form of a Minkowskian space, in the case of a ordinary scalar field, and a Euclidean space in the case where the scalar field is of phantom type. Also, the dynamics of the $(x, y)$ system is described by a Lagrangian which is a diagonal quadratic form with constant coefficients. The existence of Noether symmetry implies that the Lie derivative of this Lagrangian with respect to the infinitesimal generator of the desired symmetry vanishes. By applying this condition to the Lagrangian of the model, we obtained the explicit form of the corresponding potential function. In the both cases of ordinary or phantom scalar field we found three distinct solutions for the potential function each of which have their own classical and quantum dynamics. In more details, in the case where the scalar is an usual one the three forms of Noether symmetric potentials are found as $V(y / x)=y^{2} /\left(y^{2}-x^{2}\right), V(y / x)=x^{2} /\left(x^{2}-y^{2}\right)$ and $V=$ cons. $=1$. In the first two cases one of the variables has a cyclic dynamics while the other behaves linearly as time progresses. Also, for a constant potential function, we saw that the system of the two variables $(x, y)$ is a two dimensional oscillator-ghost-oscillator system in which both of the variables oscillate with the same frequency. The classical trajectories of these models are also obtained and it is seen that they all represent a bounded motion in the configuration space. We have shown that all of these classical solutions have a singularity of the big-bang or big-crunch type and so to pass this issue we have dealt with the quantization of the model. As for the quantum version of these models, we obtained exact solutions of the WDW equation. The wave function of the corresponding universe consists of some branches where each may be interpreted as part of the classical trajectory. We saw that since the 
peaks of the wave function follow the classical trajectory, there seems to be good correlations between the corresponding classical and quantum cosmology. The same study for a phantom scalar field yields the potentials $V(y / x)=y^{2} /\left(x^{2}+y^{2}\right), V(y / x)=x^{2} /\left(x^{2}+y^{2}\right)$ and $V=1$. For these potentials, we solved the classical equations of motion exactly and showed that at least one of the variables behaves hyperbolically. This causes the classical trajectory in $x-y$ plane to be an unbounded path in which the moving particle (universe) tends to regions where the scale factor is large. The resulting quantum cosmology and the corresponding WDW equation in the phantom framework were also studied and analytical expressions for the wave functions of the universe were presented with good correlations with the classical trajectories.

Finally, we would like to emphasize that for an ordinary scalar field the quantum effects dominate in the region of the classical big-bang singularity, i.e., at the small values of scale factor. At the big-bang the quantum solutions bounce from a contraction phase to an expansion era. On the other hand for a phantom field the quantum effects dominate in the region of the classical large scale factor so that in this region quantum solutions fall from an expansion phase to a contraction era.

\section{References}

[1] C. Brans and R.H. Dicke, Phys. Rev. 124 (1961) 925

R.H. Dicke, Phys. Rev. 125 (1962) 2163

T. Damour and G. Esposito-Fares, Class. Quantum Grav. 9 (1992) 2093

[2] A.G. Riess, et al., Astron. J. 116 (1998) 1009

B.P. Schmidt, et al., Astrophys. J. 507 (1998) 46

W. Chakraborty and U. Debnath, Role of Brans-Dicke theory with or without selfinteracting potential in cosmic acceleration (arXiv: 0807.1776 [gr-qc])

[3] A. Linde, Particle physics and inflationary cosmology (arXiv: hep-th/0503203)

D.H. Lyth and A. Riotto, Phys. Rep. 314 (1999) 1

[4] R.R. Caldwell, R. Dave and P.J. Steinhardt, Phys. Rev. Lett. 80 (1998) 1582

T. Padmanabhan, Phys. Rep. 380 (2003) 235

M. Li, X.-D. Li, S. Wang and Y. Wang, Dark energy (arXiv:1103.5870 [astro-ph.CO])

[5] Y. Fujii and K.-I. Maeda, The Scalar-Tensor Theory of Gravitation (Cambridge University Press, Cambridge, 2003)

[6] E. Komatsu, et al., Astrophys. J. Suppl. 180 (2009) 330

[7] L. Amendola and S. Tsujikawa, Dark Energy, Theory and Observations (Cambridge University Press, Cambridge, 2010)

E. Elizalde, S. Nojiri and S.D. Odintsov, Phys. Rev. D 70 (2004) 043539 (arXiv: hep-th/0405034)

R.R. Caldwell, M. Kamionkowski and N.N. Weinberg, Phys. Rev. Lett. 91 (2003) 071301 (arXiv: astro-ph/0302506)

M.P. Dąbrowski, T. Stachowiak and M. Szydłowski, Phys. Rev. D 68 (2003) 103519 (arXiv: hep-th/0307128)

[8] S.M. Carroll, M. Hoffman and M. Trodden, Phys. Rev. D 68 (2003) 023509 (arXiv: astro$\mathrm{ph} / 0301273)$

P. Singh, M. Sami and N. Dadhish, Phys. Rev. D 68 (2003) 023522 (arXiv: hep-th/0305110)

M. Sami and A. Toporenski, Mod. Phys. Lett. A 19 (2004) 1509 (arXiv: gr-qc/0312009)

M.P. Dąbrowski, C. Kiefer and B. Sandhöfer, Phys. Rev. D 74 (2006) 044022 (arXiv: hepth/0605229) 
[9] S. Capozziello, G. Marmo, C. Rubano and P. Scudellaro, Int. J. Mod. Phys. D 6 (1997) 491 (arXiv: gr-qc/9606050)

S. Capozziello, S. Nesseris and L. Perivolaropoulos, J. Cosmol. Astropart. Phys. JCAP 0712 (2007) 009 (arXiv: 0705.3586 [astro-ph])

S. Capozziello and A. De Felice, J. Cosmol. Astropart. Phys. JCAP 0808 (2008) 016 (arXiv: 0804.2163 [gr-qc])

[10] S. Capozziello, A. Stabile and A. Troisi, Class. Quantum Grav. 24 (2007) 2153 (arXiv: gr-qc/ 0703067)

A.K. Sanyal, B. Modak, C. Rubano and E. Piedipalumbo, Gen. Rel. Grav. 37 (2005) 407 (arXiv: astro-ph/0310610)

A.K. Sanyal, Phys. Lett. B 524 (2002) 177 (arXiv: gr-qc/0107053)

B. Vakili, N. Khosravi and H.R. Sepangi, Class. Quantum Grav. 24 (2007) 931 (arXiv: gr-qc/ 0701075)

B. Vakili, Phys. Lett. B 664 (2008) 16 (arXiv: 0804.3449 [gr-qc])

B. Vakili, Ann. Phys. (Berlin) 19 (2010) 359 (arXiv: 1001.2959 [gr-qc])

Y. Zhang, Y.-G. Gong and Z.-H. Zhu, Phys. Lett. B (arXiv: 0912.0067 [hep-ph])

S. Capozziello, E. Piedipalumbo, C. Rubano and P. Scudellaro, Phys. Rev. D 80 (2009) 104030 (arXiv: 0908.2362 [astro-ph.CO])

C. Rubano and P. Scudellaro, Gen. Rel. Grav. 34 (2002) 307

M. Demianski, C. Rubano and C. Tortora, Astron. Astrophys. 431 (2005) 27

M. Roshan and F. Shojai, Phys. Lett. B 668 (2008) 238 (arXiv: 0809.1272 [gr-qc])

M. Jamil, F.M. Mahomed and D. Momeni, Phys. Lett. B 702 (2011) 315 (arXiv: 1105.2610 [physics. gen-ph])

[11] S. Capozziello and G. Lambiase, Gen. Rel. Grav. 32 (2000) 673 (arXiv: gr-qc/9912083)

[12] B.S. DeWitt, Phys. Rev. 160 (1967) 1113

S.W. Hawking and D.N. Page, Nucl. Phys. B 264 (1986) 185

D. Wiltshire, An introduction to quantum cosmology (arXiv: gr-qc/0101003)

J.J. Halliwell, Introductory lectures on quantum cosmology (arXiv: 0909.2566 [gr-qc])

C. Kiefer, Quantum Gravity (Oxford University Press, New York, 2007)

[13] B. Vakili, Phys. Lett. B 669 (2008) 206 (arXiv: 0809.4591 [gr-qc])

[14] S. Basilakos, M. Tsamparlis and A. Paliathanasis, Phys. Rev. D 83 (2011) 103512 (arXiv: 1104.2980 [astro-ph.CO])

M. Tsamparlis and A. Paliathanasis, J. Phys. A 44 (2011) 175202 (arXiv: 1101.5771 [math-ph])

[15] A. Vilenkin, Phys. Rev. D 37 (1988) 888

[16] N.A. Lemos, J. Math. Phys. 37 (1996) 1449 (arXiv: gr-qc/9511082)

[17] M. Bojowald and A. Tsobanjan, Class. Quantum Grav. 27 (2010) 145004 (arXiv: 0911.4950 [gr-qc])

[18] C. Rovelli, Phys. Rev. D 42 (1990) 2683

[19] M. Abramowitz and I.A. Stegun, Handbook of Mathematical Functions (New York: Dover 1972)

[20] B. Vakili, Phys. Rev. D 83 (2011) 103505 (arXiv: 1104.1163 [gr-qc]) 